\title{
Rediseño e implementación de un curso de psicología médica desde metodología docente presencial, a una semipresencial. Curso de psicología médica en formato $b$-learning
}

\author{
Redesign and implementation of a medical psychology course \\ from face to face to blended learning methodology
}

Bryan Vargas Gajardo', Vicente Gonzalez Isla', Constanza Orozco Soto', José Pinedo Palacios², Jaime Santander Toro².

\section{Resumen}

Introducción: el curso mínimo de psicología médica tiene como propósito introducir y sensibilizar a los estudiantes en temas psicológicos básicos relevantes para la comprensión y desarrollo del ejercicio de la medicina. Entre las últimas evaluaciones de estudiantes de la Pontificia Universidad Católica, destacan comentarios negativos sobre las extensas jornadas de clases y la poca flexibilidad en la entrega de material de estudio. Métodos: se propuso implementar una metodología de clases b-learning, permitiendo al estudiante auto gestionar su tiempo y recibir los contenidos a distancia. Se evaluó posteriormente la aceptación de la nueva metodología a través de encuestas de satisfacción y se evaluó comparativamente el efecto de metodología en las calificaciones del alumnado. Resultados: se obtuvo resultados favorables con un promedio global al curso de 6,23 \pm 0,67 para el año 2016 y 6,38 \pm 0,49 para el 2017, y específicamente para la metodología b-learning de 6,03 \pm 0,97 para el 2016, y para el $20176,41 \pm 0,63$. Conclusión: Se concluye que la modalidad de $b$-learning es una metodología de aprendizaje valorada positivamente por los alumnos con repercusión favorable en sus resultados académicos.

Palabras clave: b-learning; clases online; medios audiovisuales; educación médica.

\begin{abstract}
Introduction: the minimum course of Medical Psychology aims to introduce and to make students aware of psychological issues relevant to the understanding and development of the medical practice. Between the last evaluations made by students before 2016, negative comments about the extensive class schedules stand out. Methods: It was proposed to implement a b-learning class methodology, allowing the student to self-manage their time and receive the contents remotely. We evaluated the acceptance of the new methodology through satisfaction surveys, and we evaluated the effect of this methodology on the student's qualifications. Results: favorable results were obtained with a global average to the course of $6.23 \pm 0.67$ for the year 2016 and $6.38 \pm 0.49$ for 2017, and specifically for the b-learning methodology, $6.03 \pm 0.97$ for 2016 and $6.41 \pm 0.63$ for 2017 . Conclusion: We conclude that the b-learning modality is a learning methodology valued positively by the students with a positive increase in their academic results.
\end{abstract}

Keywords: instructional design; b-learning; online courses; audiovisual media; medical education

Fecha de envío: 28 de abril de 2020 - Fecha de aceptación: 11 de junio de 2020

\section{Introducción}

El término de psicología médica fue acuñado hacia la mitad del siglo XIX, en un momento histórico en que la perspectiva humanística estaba integralmente ligada a los principios científicos, perspectiva que se ha ido adaptado a los tiempos presentes donde el quehacer científico ha cobrado más relevancia en detrimento de las consideraciones humanísticas (Rossmanith, 1990). 
Un estudio publicado el año 2012 en Chile reveló que un 83,3\% de las escuelas de medicina del país incorporan en su plan de estudios un ramo orientado a la psicología médica. Esto demuestra la importancia adjudicada a esta disciplina en la formación de médicos generales, disciplina que entrega, dadas sus características, herramientas indispensables para el quehacer médico diario (Santander et al., 2012)

Tomando en cuenta la necesidad de que el estudiante de medicina pueda administrar mejor sus tiempos de estudio y de tener más acceso a fuentes de aprendizaje, y dadas las frecuentes evaluaciones negativas hacia el curso referidas casi exclusivamente a la extensión del mismo en formato presencial, se propuso rediseñar el curso de psicología médica desde un modalidad presencial tradicional, a una modalidad semipresencial blended learning o, en adelante, b-learning, que considera clases online. Lo denominado e-learning o aprendizaje mediado por recursos electrónicos y redes) y talleres presenciales, buscando seguir con la tendencia mundial en la búsqueda de metodologías de aprendizaje que optimicen el tiempo de estudio; siendo una de las más novedosas y con mayor potencial de expansión el aprendizaje en línea (Knutson et al., 2006; Reinoso et al., 2012). El siguiente estudio propone evaluar el impacto desde el punto de vista de resultados académicos y percepción del alumnado ante el rediseño descrito hacia una modalidad semipresencial.

En general, $b$-learning se define como la combinación de clases en modalidad online (utilizando recursos tecnológicos) y encuentros presenciales de diversa naturaleza, favoreciendo un acercamiento flexible a la educación, beneficiándose en entregar contenido multimedia y presencial para mejorar los resultados del aprendizaje y abaratar costos de infraestructura y aplicación. Es decir, se trata de un modelo ecléctico compuesto por instrucción presencial y funcionalidades del aprendizaje electrónico o e-learning, cuya finalidad es potenciar las fortalezas y disminuir las limitaciones de ambas modalidades. Este modelo permite permanecer menos tiempo en el aula, propicia un potencial ahorro de espacios físicos e incrementa la participación de los estudiantes como responsables de su propio aprendizaje entre otros beneficios (Redi-Young, 2003).

Este "aprendizaje mezclado" sigue una tendencia con una marcada raíz procedente del campo de la psicología escolar en la que destaca el término "aprendizaje" como continuo que no se limita al tiempo en el aula, y que favorece estrategias activas, autónomas e independientes en el avance del aprendizaje, algo que tampoco es nuevo; las clases tradicionales de las últimas décadas han estado combinando laos encuentro magistrales con los ejercicios, los estudios de caso, juegos de rol y las grabaciones de vídeo y audio en un intento por hacerlas más atractivas y reforzar el proceso de aprendizaje (Brodsky, 2003; Bartolomé, 2004).

Este tipo de técnicas, se ha visto, cuenta con importantes resultados académicos en términos de calificaciones y logro de objetivos curriculares, validados en diferentes escenarios y con experiencias en asignaturas diversas como semiología médica en Chile (Gonzalez et al., 2015) para estudiantes de medicina, Anatomía Humana para estudiantes de primer año de medicina en Brasil (Pereira et al., 2007), enseñanza del inglés en niños de primaria en Palestina (Keshta et al., 2013) e incluso, cursos de entrenamiento de Anestesia para residentes de primer año en Francia (Marchalot et al., 2018).

El diseño de cualquier curso de b-learning no sigue una receta única, sino que debe realizarse de acuerdo al enfoque, que puede basarse en:

a. Habilidades: combina el aprendizaje a paso propio: clases online, documentos, libros, etc., con apoyo al aprendiz por medio de interacciones con el tutor a través de foros, e mail o sesiones presenciales guiadas.

b. Actitudes: se aproxima al aprendizaje combinando sesiones presenciales y laboratorios de aprendizaje guiados por el instructor, con discusiones facilitadas por la tecnología como foros o aulas virtuales

c. Competencias: Se aproxima al aprendizaje combinando tutorías basadas en la tecnología y en el "aprender haciendo" junto a expertos.

En este rediseño particular, los contenidos fueron entregados a través de clases en formato e-learning (profesor en cámara y otros medios audiovisuales), y se incluyeron encuentros presenciales para desarrollar otras competencias y profundizar lo estudiado previamente; como se ve, se prefirió un enfoque basado en Habilidades, modelo que se decidió considerando combinando la presencia de:

a. Estudiante de medicina: y su manifiesta necesidad de manejar sus tiempos de aprendizaje

b. Clases online, cuyo formato será detallado en el cuerpo de este artículo.

c. Encuentros cara a cara guiados por el tutor, a modo de conversatorios periódicos para aclarar dudas y realizar talleres con "pacientes simulados" donde los alumnos pudieran poner en práctica las premisas y conocimientos tratados en las clases online. 
La repercusión esperada de esta modalidad consistía en favorecer en el alumnado su autonomía, el manejo responsable de los tiempos y el autoaprendizaje (Adkins, 2007), considerando además que los mismos estudiantes tienen una percepción positiva sobre la realización de cursos semipresenciales (Rosales et al., 2014) y que las experiencias en otros centros latinoamericanos ha demostrado impacto estadísticamente significativo en datos duros de aprendizaje (Espinoza et al., 2016).

\section{Materiales y Métodos}

\section{Implementación del curso}

En primer lugar, la implementación del curso se dividió en una fase de diseño, en la cual se creó el programa del curso completamente orientado en un formato $b$-learning. Para ello, se revisó el programa del curso tradicional (objetivos y contenidos), y se definieron las clases que serían adaptadas al formato online, y las que se realizarían en modalidad presencial; con lo cual se tendría un curso completo en formato b-learning. A continuación, tanto para las clases en formato online como las presenciales, se definieron los recursos pedagógicos necesarios que permitirían entregar los contenidos y lograr los objetivos de aprendizaje. Luego, se redactó el libreto para las clases online. Terminado el diseño y el libreto, se procedió a grabar cada una de las clases y seleccionar los recursos audiovisuales para armar la presentación final en la fase de producción.

Para la fase de grabación y edición de medios audiovisuales, se contó con un equipo de 5 estudiantes expertos en tecnología multimedial. Se elaboraron, entonces, un total de 12 clases en formato online para los temas: procesos afectivos, procesos cognitivos, neurobiología de procesos afectivos y cognitivos, psicología y medicina evolucionaria, influencia de la personalidad en la práctica médica, ciclo vital y duelo, identidad, vocación y profesionalismo, modelos de cambio y determinantes sociales de la salud.

Se utilizó la plataforma Articulate (Schwartz, 2016) para la realización de dichas clases, con un formato estándar para cada presentación que incluía un video del profesor del curso dictando la clase, la presentación de los contenidos $y$, en caso de que ameritara, un video extraído de fuentes diversas (series de TV, películas, cultura popular) para reforzar, ejemplificar o proponer un análisis. Estos espacios fueron Ilamados "cápsulas".

Las clases, intentado de la manera más efectiva emular una clase lectiva real (aunque, en este caso, asincrónica) constaban de una presentación estándar y de un pequeño video al costado inferior de la pantalla, donde aparece el profesor dictando el material instruccional que se va desplegando y cuyo avance puede pasar, retroceder o avanzar el alumno acorde a sus necesidades. Dichas clases eran subidas a la plataforma web oficial del curso de acuerdo a un calendario previamente diseñado y duraban en promedio 1 semana para poder ser revisadas por el alumnado, con una duración de entre 1 a 3 horas (promedio 1,7 horas cronológicas) por sesión completa, acorde a lo exigido en el currículum del alumno de medicina.

\section{Evaluación del curso}

Para evaluar los contenidos entregados se sometió a los alumnos a cuestionarios online, con preguntas de desarrollo que contemplaban análisis crítico, definición de conceptos, discusión de casos, etc., que fueron subidas a la plataforma web del curso a primera hora del día calendarizado para dicha evaluación, dándose un plazo de 1 hora para su realización.

\section{Impacto de la intervención}

Para medir el impacto de la implementación de la modalidad b-learning, al término de la primera y segunda cohorte en que se impartió el curso rediseñado de psicología médica, se aplicó un cuestionario de satisfacción con la experiencia. En esta encuesta se evaluó la metodología del curso, las clases online (formato e-learning) y los medios audiovisuales (profesor en cámara, cápsulas de videos, etc); el alumno debía determinar su grado de acuerdo, siendo 1 muy en desacuerdo y 4 muy de acuerdo. Además, se solicitó calificar tanto la metodología y el curso globalmente con una nota de 1 a 7. Se realizó posteriormente un análisis de validez de contenido, muestreo y constructo mediante un análisis factorial exploratorio con rotación Varimax. Se seleccionó a los factores de acuerdo al criterio de Kaiser-Guttman, que incluye a aquellos con eigenvalue $>1$ (Buja \& Eyuboglu, 1992). Se utilizó, además, el criterio de Cattell (Cattell, 1966) estableciendo el punto de inflexión en el gráfico de sedimentación como valor de corte, considerando todos los factores sobre el mismo. Se midió además la fiabilidad de los resultados de la encuesta, en base al análisis de consistencia con alfa de Cronbach, considerando como buena consistencia un resultado superior a 0,8 (Cronbach, 1951). Además, se aplicó la encuesta tradicional de evaluación de cursos, que se realiza en base a recomendaciones del Centro de Educación Médica de la Facultad de Medicina de la Pontificia Universidad Católica de Chile.

\section{Resultados}

Para validar la encuesta de satisfacción el año 2016, se realizó un análisis factorial en el que destaca una estructura con 2 factores, de acuerdo al criterio de Kaiser-Guttman. El primer factor (que 
incluye las preguntas $1 \mathrm{~A}, 3 \mathrm{~A}, 1 \mathrm{~B}, 2 \mathrm{~B}, 3 \mathrm{~B}, 4 \mathrm{~B}$ y $5 \mathrm{~B}$ ) dio cuenta de un $50,6 \%$ de la varianza, mientras que el conjunto de éste y el segundo factor (que contiene la pregunta $2 \mathrm{~A}$ ), explican el $65,9 \%$ de la varianza total.

Se evaluó además la consistencia de la encuesta, mediante el alfa de Cronbach, que resultó en 0,84 incluyendo todas las preguntas y 0,87 excluyendo la pregunta $3 \mathrm{~A}$. Cabe destacar que ambos resultados dan cuenta de una buena consistencia. Se sometió a la encuesta del 2017 al mismo análisis.

Para el 2017, el análisis resulta en una estructura de 2 factores, con un primer factor que incluye preguntas $(1 \mathrm{~A}, 3 \mathrm{~A}, 1 \mathrm{~B}, 2 \mathrm{~B}, 3 \mathrm{~B}, 4 \mathrm{~B}$ y $5 B$ ) y un segundo factor (con la pregunta $2 A$ ), explicando $45,7 \%$ y $14,6 \%$ de la varianza total respectivamente, sumando entre ambos un 60,3\%. El valor del alfa de Cronbach fue de 0,8 incluyendo todas las preguntas y 0,833 al excluir la pregunta $3 \mathrm{~A}$.

El total de los alumnos que realizaron el curso en el periodo 20142017 fue de 591, de los cuales 112 lo cursaron el año 2014, 244 el año 2015 (año en que cursaron paralelamente 2 generaciones la asignatura), 112 el 2016 y 123 el 2017. Del total de los alumnos la encuesta de satisfacción fue contestada por 107 alumnos el año 2014, 124 en el curso de primer año el 2015 y 115 en el curso de segundo año, 81 el 2016 y 113 el 2017.

Respecto a la encuesta específica para el curso de psicología médica, tanto en los años 2016 y 2017, todas las aseveraciones tuvieron un alto grado de acuerdo (entre 2,91 y 3,77 el 2016, y entre 3,11 y 3,82 el 2017). Los resultados para ambos periodos se muestran en la tabla 2.

Las aseveraciones con mayor grado de acuerdo fueron "El apoyo de cápsulas de ejemplos tomados del cine, televisión y documentales, fue un buen apoyo para comprender de mejor manera los contenidos" $y$ "La utilidad de la metodología para manejar sus tiempos de estudio". Las aseveraciones con menor grado de acuerdo fueron "Me resultó fácil acostumbrar a esta modalidad de aprendizaje semipresencial" para ambos años.

La calificación promedio global del curso para el año 2016 fue de $6,23 \pm 0,67$ y $6,38 \pm 0,49$ para el 2017 , mientras que el promedio para b-learning fue de 6,03 $\pm 0,97$ para el 2016, y para el $20176,41 \pm 0,63$. Además, se obtuvo los resultados de la tradicional encuesta realizada por el Departamento de Educación Médica de la facultad, en donde se califica al curso de forma global, esto permite tener una estimación respecto a la opinión pre y post implementación del curso rediseñado, los resultados se muestran en la tabla 3.
Tabla 1: Preguntas de encuesta de satisfacción y respuestas por grados de acuerdo.

Con respecto a las clases videadas: Malo (1), regular (2), bueno (3), muy bueno (4)

1A: Las clases videadas con profesor en cámara y apoyo con PPT sincronizado, me pareció un recurso:

2A:El apoyo de cápsulas de ejemplos tomados del cine, televisión y documentales, fue un buen apoyo para comprender de mejor manera los contenidos: 3A: En general, la entrega de contenidos a través de clases e-learning (on line, a distancia) me pareció una metodología:

Con respecto a tu impresión global del curso, la metodología b-learning (semipresencial) de este curso de Psicología Médica, me pareció una buena alternativa para: Muy en desacuerdo (1), en desacuerdo (2), de acuerdo (3), muy de acuerdo (4)

1B: Manejar mis propios tiempos de aprendizaje

2B: Acceder a los contenidos de acuerdo a mis intereses y motivaciones 3B: La metodología b-learning (semipresencial) es una ventaja sobre las metodologías presenciales tradicionales

4B: La mezcla de clases on-line, encuentros presenciales, tutorías a distancia y trabajos de aprendizaje para la casa me pareció adecuada 5B: Me resultó difícil acostumbrarme a esta modalidad de aprendizaje semipresencial

Tabla 2: Aseveraciones en encuesta de satisfacción (grados de acuerdo de 1 a 4), y calificación al curso y metodología de 1 a 7.

\begin{tabular}{l|c|c}
\hline $\begin{array}{l}\text { Preguntas encuesta de satisfacción } \\
\text { (grados de acuerdo de 1 a 4) }\end{array}$ & $\begin{array}{c}\text { Promedios } \mathbf{\text { DS }} \\
\mathbf{( 2 0 1 6 )}\end{array}$ & $\begin{array}{c}\text { Promedios } \pm \\
\text { DS (2017) }\end{array}$ \\
\hline $\begin{array}{l}\text { Las clases videadas con profesor en } \\
\text { cámara y apoyo con PPT sincronizado, } \\
\text { me pareció un recurso: }\end{array}$ & $3,25 \pm 0,92$ & $3,38 \pm 0,64$ \\
\hline $\begin{array}{l}\text { El apoyo de cápsulas de ejemplos toma- } \\
\text { dos del cine, televisióny documentales, } \\
\text { fue un buen apoyo para comprender } \\
\text { de mejor manera los contenidos: }\end{array}$ & $3.77 \pm 0,55$ & $3.82 \pm 0,48$ \\
\hline $\begin{array}{l}\text { En general, la entrega de contenidos a } \\
\text { través de clases e-learning (on line, a } \\
\text { distancia) me pareció una metodología: }\end{array}$ & $3.17 \pm 0,88$ & $3,14 \pm 0,87$ \\
\hline $\begin{array}{l}\text { La metodología b-learning permitió } \\
\text { mejorar mis propios tiempos de } \\
\text { aprendizaje: }\end{array}$ & $3.47 \pm 0,82$ & $3.43 \pm 0,69$ \\
\hline $\begin{array}{l}\text { Acceder a los contenidos de acuerdo } \\
\text { a mis intereses y motivaciones }\end{array}$ & $3.46 \pm 0,84$ & $3.38 \pm 0,72$ \\
\hline $\begin{array}{l}\text { La metodología b-learning (semipre- } \\
\text { sencial) es una ventaja sobre las me- } \\
\text { todologías presenciales tradicionales: }\end{array}$ & $3.15 \pm 0,88$ & $3.23 \pm 0,83$ \\
\hline $\begin{array}{l}\text { La mezcla de clases on-line, encuentros } \\
\text { presenciales, tutorías a distancia y } \\
\text { trabajos de aprendizaje para la casa } \\
\text { me pareció adecuada }\end{array}$ & $3,21 \pm 0,9$ & $3,36 \pm 0,79$ \\
\hline $\begin{array}{l}\text { Me resultó fácil acostumbrarme } \\
\text { a esta modalidad de aprendizaje } \\
\text { semipresencial: }\end{array}$ & $2,91 \pm 1,10$ & $3.11 \pm 1,07$ \\
\hline $\begin{array}{l}\text { Calificación de 1 a 7 } \\
\text { ¿Qué nota global le pondría a este } \\
\text { curso? (contenidos y aprendizaje): }\end{array}$ & $6,23 \pm 0,67$ & $6,38 \pm 0,49$ \\
\hline $\begin{array}{l}\text { ¿Qué nota global le pondría a este } \\
\text { curso? (metodología b-learning): }\end{array}$ & $6,03 \pm 0,97$ & $6,41 \pm 0,63$ \\
\hline
\end{tabular}


Tabla 3: Nota global curso pre y post implementación.

\begin{tabular}{l|l}
\hline Año & Promedios \pm DS \\
\hline 2014 & $6,1 \pm 0,76$ \\
\hline $2015-1^{\circ}$ año & $5,87 \pm 0,95$ \\
\hline $2015-2^{\circ}$ año & $5,74 \pm 1,29$ \\
\hline 2017 & $6,48 \pm 0,67$ \\
\hline
\end{tabular}

\section{Discusión}

La realización del curso de psicología médica en formato $b$-learning tuvo resultados prometedores para la educación médica. La evaluación general de los alumnos respecto al curso de psicología manifestó importantes avances, viéndose reflejado en las respuestas a la encuesta aplicada, validada mediante los métodos estadísticos previamente descritos.

Dentro de los resultados del cuestionario, cabe destacar como las dos afirmaciones con mayor acuerdo aquellas referidas a la utilización de elementos audiovisuales extra que "reforzaran los contenidos" y a la oportunidad de "autoadministrar los tiempos de aprendizaje". Además, a través de la evaluación aplicada por el Centro de Educación Médica, se observa una mejor calificación global del curso el año 2017 (b-learning) versus 2014-2015 (presencial); considerando que los contenidos se mantuvieron constantes inter-generacionalmente, se le puede atribuir a la modalidad semipresencial, que incluye clases formato online y encuentros presenciales a modo de reforzamiento y profundización, esta mejora académica. Resulta interesante el valor que le da el estudiante a la inclusión de material que le resulte llamativo y familiar en el proceso de aprendizaje. De igual modo, se resalta el gran valor que el estudiante le asigna a la flexibilidad horaria y a la capacidad de distribuir su tiempo libre, lo que parece ser clave tanto en la propuesta de la nueva malla curricular como en el proceso de adaptación de un estudiante en transición a la vida universitaria. Esto da paso para una nueva configuración de la interacción docente alumno, donde principalmente se constituye por un espacio de resolución de dudas y no meramente lectivo, permitiendo que el alumno gaste menos tiempo en la sala de clase sin que esto perjudique la entrega de contenidos mínimos. Se podría pensar que en la histórica mala evaluación del curso subyace más bien falta de interés y relevancia del alumnado para con esta asignatura como principal causa. Si bien, podría considerarse un argumento válido, este, finalmente, contribuye a sostener aún más la necesidad de optimizar la forma en que los contendidos menos valorados por el alumnado son entregados, de manera que resulte eficiente tanto para ellos como para la misma institución, y se logren los objetivos de aprendizaje en común. De todas maneras, en contra de dicho argumento, se podrían esgrimir contestaciones de naturaleza igual de subjetivas, pero en base a nuestro estudio, al haber mantenido la cantidad y calidad de contenidos y sin haber diferencias sustanciales en las calificaciones - punto verdaderamente esencial en la apreciación del estudiante de medicina-, se le puede atribuir con bastante seguridad la mejoría en la percepción al medio en el que se desarrolla el curso. Sin embargo, no es objetivo de este estudio entrar en cavilaciones subjetivas sobre este punto.

Cabe mencionar que durante la realización de las evaluaciones online se pesquisaron casos aislados de comportamientos irregulares por parte de los alumnos referidos a copia flagrante y evidente de contenidos; queda abierto al debate sobre la madurez, capacidad e integridad de un alumno de primer año de medicina para realizar este tipo de actividades formales, pudiendo a su vez tratarse de casos puntuales ya que, evidentemente, no fue la tónica del curso.

Nos parece bastante evidente que la integración de material multimedia: videos, textos, imágenes, audio, y la ubiquidad del proceso educativo son características que solo reciamente han podido aprovecharse en pos del desarrollo de modelos educativos más vinculantes y holísticos. Lo que presupone oportunidades, pero también desafíos. Datos relativamente recientes de la Unión Europea muestran que entre el 50 al $80 \%$ de los estudiantes en dichos países nunca han usado textos digitales, softwares de ejercicios, podcast, juego de simulación, esto sumado además a la reticencia de los profesores a nivel al menos primario y secundario de ser capaces de utilizar eficientemente estos elementos (liyoshi et al., 2016.); pero en una población estudiantil que ha creciendo rápidamente, se hace imprescindible adaptar los métodos tradicionales de aprendizaje o al menos ofrecer un mix cara a cara y online, al modo del consabido b-learning. Este desafío también es un desafío para los científicos y expertos en educación, puesto que hay relativamente poco conocimiento sobre cómo usar las redes sociales y los elementos tecnológicos para desarrollar una educación más significativa, efectiva y atractiva, versus el amplísimo conocimiento respecto a las potencialidades de la tecnología como canal de entretención y esparcimiento.

Se reconocen algunas dificultades en la enseñanza de la psicología aun en procesos semipresenciales, dificultades ya enunciadas por Benbassat et al, (2003), en torno a tres puntos esenciales: a) la necesidad priorizar los objetivos de aprendizaje de acuerdo con competencias clínicas; b) la importancia del currículo integración siguiendo el modelo biopsicosocial y c) la necesidad de maestros entrenados en la aplicación de ciencias del comportamiento dentro de la práctica médica. La dificultad de los estudiantes en aceptar estas disciplinas eventualmente está justificada por la 
ausencia de pautas en la mayoría de las escuelas de medicina. Por lo tanto, se requiere un esfuerzo para aumentar la cantidad y calidad de la enseñanza de habilidades sociales en la educación y formación de médico (Isaac \& Rief, 2009). También es necesario cambiar la cultura médica, teniendo en cuenta las diversas facetas y normativas carácter de los paradigmas sobre la salud, la enfermedad y la salud practicar, o al menos ganar la capacidad de cuestionar estos paradigmas y desarrollar pensamiento crítico (Wong G, 2013).

\section{Fuentes de financiamiento}

Departamento de Psiquiatría, Pontificia Universidad Católica de Chile. Vicerrectoría académica, a través de proyecto FONDEDOC 2016, Pontificia Unviersidad Católica de Chile.

\section{Contribuciones y conflictos declarados por los autores}

Los autores declaramos no tener conflictos de interés.

\section{Referencias}

Adkins S. (2007) Waves of Innovation: From Open Source to Open Learning. Learning Circuits 23

Azeiteiro, U., Leal Filho, W., \& Caeiro, S. (2014). E-learning and education for sustainability (pp. 1-290). Peter Lang.

Benbassat J, Baumal R, Borkan JM \& Ber R. (2003). Overcoming barriers to teaching the behavioral and social sciences to medical students. Academic Medicine 78, 372-380.

Buja A \& Eyuboglu N. (1992). Remarks on parallel analysis. Multivariate behavioral research 27, 509-540.

Cattell RB. (1966). The scree test for the number of factors. Multivariate behavioral research 1, 245-27

Cronbach LJ. (1951). Coefficient alpha and the internal structure of tests. psychometrika 16, 297-334.

Espinoza E, Coveña D, Andrade H, Segarra J \& Naranjo JR. (2016). Evaluación del uso del programa B learning en estudiantes de Medicina, sobre tratamiento inicial y prevención de trauma en niños. Cuenca 2015. Uniandes Episteme 3, 360-371.

González, A., Vargas, B., González, V., Reyes, I., \& Sarfatis, A. (2016). Módulos interactivos en línea de semiología médica: Una herramienta para estandarizar el aprendizaje clínico. Revista médica de Chile 144, 1605-1611.
Isaac M \& Rief W. (2009). Role of behavioural and social sciences in medical education. Curr Opin Psychiatry 22, 184-187.

liyoshi, T \& Kumar, MSV. (2016). Opening Up Education (p. 504). The MIT Press.

Keshta, AS \& Harb II. (2013). The effectiveness of a blended learning program on developing Palestinian tenth graders'English writing skills. Education Journal 2 , 208-221.

Knutson D, Cain T, Hurtubise L \& Kreger C. (2006). Lessons learned: developing e-learning to teach physical examination. The Clinical Teacher 3, 163-169

Marchalot A, Dureuil B, Veber, B, Fellahi, JL, Hanouz, JL, Dupont H, Lorne E, Gerard JL, Vincent \& Compère V. (2018). Effectiveness of a blended learning course and flipped classroom in first year anaesthesia training. Anaesthesia Critical Care \& Pain Medicine 37, 411-415.

Pereira JA, Pleguezuelos, E, Merí, A, Molina-Ros A, Molina-Tomás MC \& Masdeu C. (2007). Effectiveness of using blended learning strategies for teaching and learning human anatomy. Medical education 41, 189-195.

Reid-Young A. (2003). The key of successful e-learning is b-learning. $\mathrm{HCl}$, Journal of Information Developlment. Retrieved Febryary 7, 2006, Accedido en: fttp://www.hci.com.au/hcisite2/ journal/Key\%20to\%20elearning\%20is\%20blearning.html el 12 de octubre de 2019.

Reinoso T, Tamarit T \& Pérez G. (2012). La formación de recursos humanos en salud necesarios para el mundo y los paradigmas vigentes. Educación Médica Superior 26, 635-641.

Rosales G, Gómez V \& Guzmán J. (2014) Ventajas de los cursos B-learning en medicina de pregrado. Percepción de los alumnos. Revista de la Escuela de Medicina Dr José Sierra Flores 28, 81-86.

Rossmanith S. (1990). The Importance and Purpose of Medical Psychology in the Study of Medicine. Psychotherapy and Psychosomatics $\mathbf{5 3}, \mathbf{1 0 8 - 1 1 4 .}$

Santander J, Pinedo J \& Repetto P. (2012). Enseñanza de Psicología Médica en las escuelas de medicina chilenas. Revista médica de Chile 140, 946-951.

Schwartz A. (2016). Articulate Storyline. Articulate, New York.

Wong G. (2013) The curse of paradigms? Med Educ. 47, 333-41. 